IOS Press

\title{
Editorial
}

\section{Special issue on Linked Data for Health Care and the Life Sciences}

\author{
Mikel Egaña Aranguren ${ }^{\mathrm{a}}$, Jesualdo Tomás Fernández-Breis ${ }^{\mathrm{b}}$ and Michel Dumontier ${ }^{\mathrm{c}}$ \\ ${ }^{a}$ Centre for Plant Biotechnology and Genomics (CBGP), Technical University of Madrid (UPM), Spain \\ E-mail: mikel.egana.aranguren@upm.es \\ ${ }^{\mathrm{b}}$ School of Computer Science, University of Murcia (UM), Spain \\ ${ }^{\mathrm{c}}$ Department of Biology, Carleton University, Canada
}

Health Care and Life Sciences (HCLS) have long been a test-bed for the standards proposed by the W3C to build the Semantic Web ${ }^{1}$ : since HCLS is descriptive by nature and its descriptions have traditionally been produced according to ad-hoc schemas in isolated resources, HCLS offers an ideal use case for technologies like RDF${ }^{2}, \mathrm{SPARQL}^{3}$ and $\mathrm{OWL}^{4}[1,4]$. This "marriage" of the HCLS domain with semantic technologies has resulted in a collection of resources that can be regarded as an HCLS-focused working implementation of the idea of the Semantic Web: the socalled Life Sciences Semantic Web (LSSW).

As part of the process of implementing the LSSW, the HCLS community has adopted the Linked Data practices to publish information in a machine-friendly and linkable fashion [3], as a "down-to-earth" version of a prospective fully-fledged Semantic Web. This has resulted in members of the HCLS community, like the W3C HCLS Interest Group ${ }^{5}$, considerably contributing to the Linked Open Data (LOD) endeavour, with datasets like Bio2RDF [2] and Linked Open Drug Data (LODD) [5].

As the LOD network grows, producers and consumers alike are facing new challenges regarding interoperable vocabularies, filtering, graphical interfaces,

\footnotetext{
${ }^{1}$ http://www.w3.org/standards/semanticweb/

${ }^{2}$ http://www.w3.org/standards/techs/rdf

${ }^{3}$ http://www.w3.org/standards/techs/sparq

${ }^{4}$ http://www.w3.org/standards/techs/owl

${ }^{5}$ http://www.w3.org/blog/hcls/
}

best practices for dataset production, dataset quality evaluation, role of automated reasoning, etc. The aim of this special issue is to showcase some of the latest developments in the application of Linked Data in HCLS and help pave the way to solve such challenges. The issue comprises the following manuscripts: in "Applying Linked Data Approaches to Pharmacology: Architectural Decisions and Implementation" Alasdair J.G. Gray et al. describe the technical decisions made when building the Open Pharmacological Space (OPS), a drug discovery platform part of the Open PHACTS project ${ }^{6}$; in "Using the relation ontology Metarel for modelling Linked Data as multi-digraphs" Ward Blondé et al. describe Metarel, an ontology that describes class-level relations that can be used for SPARQL queries; in "A collaborative methodology for developing a semantic model for interlinking Cancer Chemoprevention linked-data sources" Dimitris Zeginis et al. describe how they used a new collaborative methodology to build a semantic model related to cancer chemoprevention.

The manuscripts went through a rigorous review process in which half of the submissions were accepted. We would like to thank the referees for their time and effort: Robert Stevens, Iker Huerga, Erick Antezana, Mari Carmen Suárez Figueroa, Mark D. Wilkinson, Andrea Splendiani, Helena Deus, Richard Boyce, Simon Jupp, Carlos Buil Aranda, Alejandro

${ }^{6}$ http://www.openphacts.org/ 
Rodríguez González, Matt-Mouley Bouamrane, Boris Villazón Terrazas, Rafael Valencia-García.

We hope that this special issue will inspire HCLS members to publish and consume HCLS related Linked Data in innovative ways, and in doing so help to build, in the long term, a more efficient LSSW that can yield new discoveries more rapidly. This should ultimately serve as an example of the utility of semantic technologies for the mainstream Web.

\section{Acknowledgements}

Mikel Egaña Aranguren is funded by the Marie Curie-COFUND Programme (FP7) of the EU. Jesualdo Tomás Fernández-Breis is funded by the Ministerio de Economía y Competitividad of Spain through grant TIN2010-21388-C02-02 and co-funded by the EU through FEDER.

\section{References}

[1] E. Antezana, M. Kuiper, and V. Mironov, Biological knowledge management: The emerging role of the Semantic Web technologies, Brief. Bioinform. 10(4) (2009), 392-407.

[2] F. Belleau, M. Nolin, N. Tourigny, P. Rigault, and J. Morissette, Bio2RDF: Towards a mashup to build bioinformatics knowledge systems, Journal of Biomedical Informatics 41(5) (2008), 706716 ,

[3] C. Bizer, T. Heath, and T. Berners-Lee, Linked Data - The story so far, International Journal on Semantic Web and Information Systems (IJSWIS) 5(3) (2009), 1-22.

[4] B.M. Good and M.D. Wilkinson, The life sciences Semantic Web is full of creeps! Brief Bioinform 7(3) (September 2006), 275-286.

[5] M. Samwald, A. Jentzsch, C. Bouton, C. Kallesoe, E. Willighagen, J. Hajagos, M. Marshall, E. Prud'hommeaux, O. Hassanzadeh, E. Pichler, and S. Stephens, Linked open drug data for pharmaceutical research and development, Journal of Cheminformatics 3(1) (2011), 19+. 